\title{
Meter Long, Homogeneous Plasma Source for Advanced Accelerator Applications
}

\author{
P. Muggli," K.A. Marsh," S. Wang," C.E. Clayton," T.C. Katsouleas," \\ and C. Joshi"
}

Unversity of Sonthem California

Los Angeles, CA 90089

"University of California, Los Angeles

Los Angcles, CA 90095

\begin{abstract}
A prototype for a 1-meter long plasma source is developed for plasma acceleration experiments. The lithium neutral vapor with a density of $2 \times 10^{15} \mathrm{~cm}^{-3}$ is ionized by a uv laser pulse and produces a plasma density of $2.6 \times 10^{14} \mathrm{~cm}^{3}$. The plasma density is limited by the available uv energy. In this prototype. the length of the neutral vapor and plasma column is $25 \mathrm{~cm}$. $\Lambda \mathrm{fter}$ ionization the plasma density decreases by a factor of two in about $12 \mu \mathrm{s}$. Interferometry in the visible on the lithium neutrals as well as $\mathrm{CO}_{2}$ laser interferometry on the plasma electrons are used as diagnostics for the plasma density.
\end{abstract}

\section{INTRODUCTION}

The latest experimental results on electron acceleration in relativistic plasma waves demonstrate energy gains up to $100 \mathrm{MeV} .^{1.2}$ The maximum energy observed in these experiments is limited by the length over which the large amplitude coherent plasma waves (wakes) can be excited. In these experiments the observed energy spread is $100 \%$ because the electrons are either self-trapped or injected in a bunch longer than a plasma period. In the LWFA scheme, gradients up to $100 \mathrm{GeV} / \mathrm{m}$ are excited over a few millimeters with a plasma density $n_{\mathrm{r}}$ in the $10^{\mathrm{r}} \mathrm{cm}^{-3}$ range. In the PBWA scheme, ${ }^{2}$ gradients of the order of $3 \mathrm{GeV} / \mathrm{m}$ are excited over $1 \mathrm{~cm}\left(n_{\mathrm{c}} \approx 10^{16} \mathrm{~cm}^{-3}\right)$. In all these experiments the energy gain $\Delta W$ is given by:

$$
\mathrm{W} \propto \boldsymbol{\varepsilon} \cdot \sqrt{\mathrm{n}_{\mathrm{e}}} \cdot \mathrm{L}
$$

where $\varepsilon=\delta n / n$, is the wake amplitude $(0.3<\varepsilon<0.5)$, and $L$ is the length over which the wake is excited. Future experiments will aim at demonstrating energy gains of the order of $1 \mathrm{GeV}$ by operating at lower plasma densities $\left(n_{r} \approx 10^{19} \mathrm{~cm}^{-3}\right)$ but over a longer length $(L \approx 1 \mathrm{~m})$.

Recently a plasma-wakefield acceleration (PWFA) experiment has been proposed $^{3}$ in which the goal is to demonstrate electron acceleration by $1 \mathrm{GeV}$ in a 1-meter long plasma. In that experiment known as E-157, the bulk of a $30 \mathrm{GeV}$ SLAC-FFTB electron bunch generates a $1 \mathrm{GeV} / \mathrm{m}$ plasma wake, and the trailing electrons of the same bunch experience the acceleration. An energy gain of $1 \mathrm{GeV}$ is in principle possible with a wake excited over a distance of 1 meter in a $n_{e} \approx 2-4 \times 10^{14} \mathrm{~cm}^{-3}$ plasma. A prototype for a 1-meter plasma source that should fulfill the requirements for the proposed experiment is described here.

CP472, Advanced Accelerator Concepts: Eighth Workshop, edited by W. Lawson, C. Bellamy, and D. Brosius

(C) 1999 The American Institute of Physics 1-56396-889-4/99/\$15.00 
The plasma source consists of a lithium ( $\mathrm{Li}$ ) heat-pipe oven which produces a $\mathrm{Li}$ vapor density in the $10^{15} \mathrm{~cm}^{-3}$ range over $25 \mathrm{~cm}$. The vapor is ionized by a uv laser pulse to a plasma density in the $10^{-14} \mathrm{~cm}^{-3}$ range.

\section{PLASMA REQUIREMENTS}

The plasma parameters required for the E-157 experiments, listed in Table 1. can be deduced for the SLAC-FFTB beam parameters, and from numerical PIC simulations." They are as follows (Table 2). a) An electron plasma density $n$ adjustable around $4 \times 10^{1+} \mathrm{cm}^{-3}$ over 1 meter to optimize the energy gain and the number of accelerated particles. Larger energy gains can be achieved at lower plasma density, but in this case less particles would be accelerated. b) A density variation $\delta n_{c} / n_{c}<25 \%$ over $1 \mathrm{~m}$ to avoid dephasing between the accelerated particles and the plasma wake. $\mathrm{c}$ ) A plasma diameter larger than $600 \mu \mathrm{m}$ to avoid the degradation of the wake amplitude caused by the excursion of the plasma electrons to outside of the plasma when blown out by the electron bunch. d) An adjustable plasma length $L$. and sharp plasma/vacuum boundaries to match the electron bunch betatron wavelength $(\approx 40 \mathrm{~cm}$ with $\left.n=2 \times 10^{14} \mathrm{~cm}^{-2}\right)$. e) A low atomic number $Z$ for the gas/plasma nuclei, and a large fractional ionization $\left(n_{l} / n_{l}>15 \%\right)$, to minimize the influence of impact ionization of the neutrals by the driving electron bunch. Estimates ${ }^{4}$ show that with $N_{l}=3 \times 10^{10}$ electrons about $1.6 \%$ ionization for $Z=2(\mathrm{He})$ and $4.5 \%$ for $Z=10(\mathrm{Ne})$ could be generated by this process at the locations where the beam pinches to its minimum spotsize $(\approx 0.5 \mu \mathrm{m})$.

Lithium is chosen (Table 3 ) because it can be photo-ionized by uv light (1-photon process) and has $Z=3$. Vapor pressures corresponding to neutral densities in the $10^{15} \mathrm{~cm}^{-3}$ range can be obtained at temperatures around $750^{\circ} \mathrm{C}$ in a heat-pipe oven.

\begin{tabular}{||l|l|l||}
\hline Number of Electrons & $\mathrm{N}_{\mathrm{e}}$ & $3.5-4.0 \times 10^{10}$ \\
\hline Initial Energy & $\mathrm{E}_{1)}$ & $30 \mathrm{GeV}$ \\
\hline Bunch Length & $\sigma_{z}$ & $0.6 \mathrm{~mm}$ \\
\hline Bunch Size & $\sigma_{\mathrm{x}}$ & $23 \mu \mathrm{m}$ \\
\hline$\left(@ 1 \times 10^{10} \mathrm{e}\right)$ & $\sigma_{\mathrm{y}}$ & $37 \mu \mathrm{m}$ \\
\hline
\end{tabular}

Table 1: Parameters for the SLACFFTB driving electron beam. ${ }^{3}$

\begin{tabular}{||l|l|l||}
\hline Plasma Density & $\mathrm{n}_{\mathrm{c}}$ & $4 \times 10^{14} \mathrm{~cm}^{-3}$ \\
\hline Length & $\mathrm{L}$ & $1 \mathrm{~m}$ \\
\hline Density Uniformity & $\delta n_{i^{\prime}} / n_{t^{\prime}}$ & $<25 \%$ \\
\hline Ionization Fraction & $n_{e} / n_{i}$ & $>15 \%$ \\
\hline Diameter & $\mathrm{d}$ & $>600 \mu \mathrm{m}$ \\
\hline
\end{tabular}

Table 2: Required plasma parameters.

\begin{tabular}{|c|c|}
\hline Li Atomic Number & 3 \\
\hline Atomic Weight & 6.94 \\
\hline Density $\left(@ 25^{\circ} \mathrm{C}\right)$ & 0.534 \\
\hline Thermal Conductivity & $0.847 \mathrm{~W} / \mathrm{cm} \mathrm{K}$ \\
\hline Heat Capacity @ $25^{\circ} \mathrm{C}$ & $3.582 \mathrm{~J} / \mathrm{g} \mathrm{K}$ \\
\hline Melting Point & $180.54^{\circ} \mathrm{C}$ \\
\hline Boiling Point @ 1 atm. & $1342{ }^{\circ} \mathrm{C}$ \\
\hline Fusion Enthalpy & $432 \mathrm{~J} / \mathrm{g}$ \\
\hline Vaporization Enthalpy & $19 \mathrm{~J} / \mathrm{g}$ \\
\hline Ionization Potential: $\mathrm{Li}^{\prime}$ & $5.392 \mathrm{eV}$ \\
\hline$: \mathrm{Li}^{\prime \prime}$ & $75.638 \mathrm{eV}$ \\
\hline
\end{tabular}

Table 3: Lithium physical characteristics. ${ }^{5}$ 


\section{HEAT-PIPE OVEN}

The heat-pipe oven (Fig. 1) consists of a stainless steel tube heated along its central part, cooled at both ends, and containing a stainless steel mesh (wick). ${ }^{6}$ The cold oven is filled with a given pressure of helium (buffer gas, $P_{\text {buffer }} \approx 200 \mathrm{mT}$ ), and contains an ingot of $\mathrm{Li}$. When heated, the Li melts and its vapor pressure increases. At some heating power, the oven reaches a temperature such that the vapor pressure of the $\mathrm{Li}$ is equal to $P_{b u j i c t}$. Above this heating power, the temperature of the $\mathrm{Li}$ does not increase. In this regime, the buffer gas at both ends of the oven contains the pure $\mathrm{Li}$ vapor to the central region of the oven. The Li pressure and temperature (i.e., density $\left.n_{0}\right)$ are fixed by the buffer pressure $\left(P_{L i}=P_{b u f f i r}\right.$ in absence of flow), while the $\mathrm{Li}$ column length is proportional to the heating power. The Li evaporates in the heated zone of the oven, and condenses at the ends. The wet wick returns the liquid $\mathrm{Li}$ toward the evaporation zone by capillary action. The prototype described here is heated over $40 \mathrm{~cm}$, the insulation is $46 \mathrm{~cm}$ long, while the distance between the cooling jackets is $52 \mathrm{~cm}$. Optical windows (quartz or $\mathrm{BaF}_{2}$ ) are located at the ends of the oven, and are in contact only with the room temperature buffer gas. They provide access for the ionizing laser pulse and for optical diagnostics of the Li vapor and plasma.
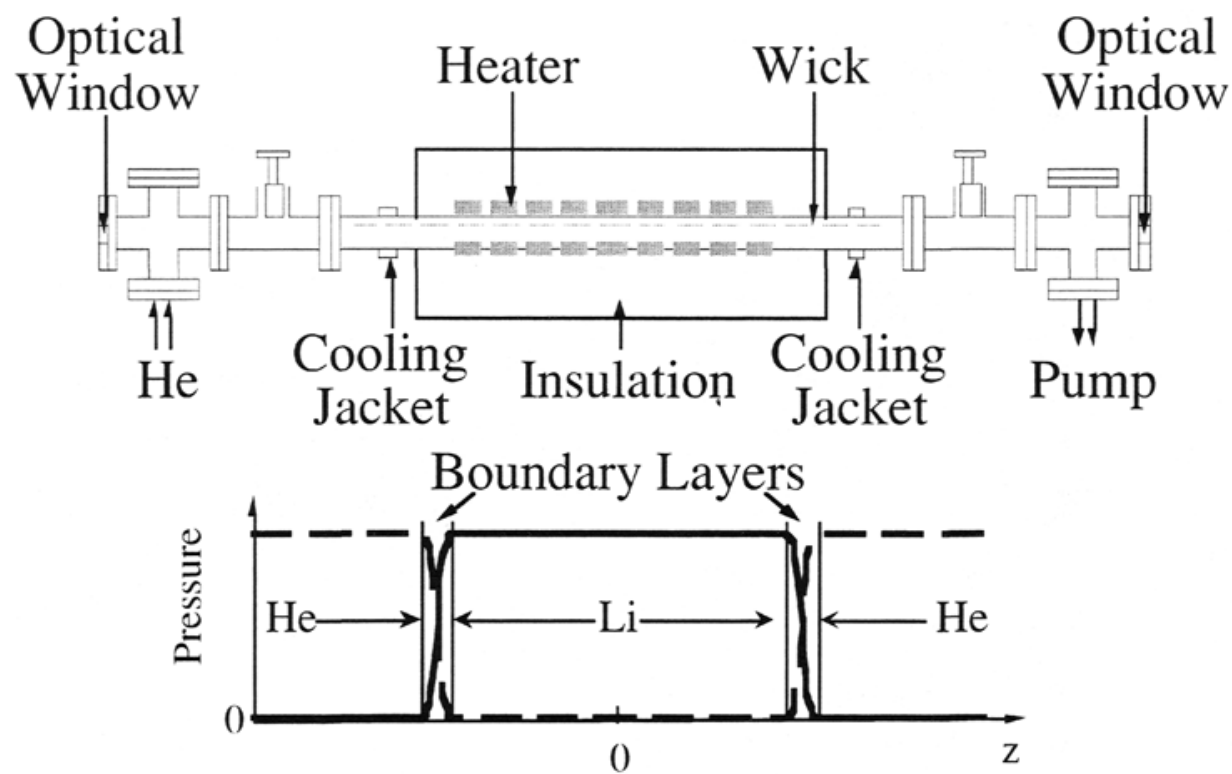

Figure 1: Schematic of the heat-pipe oven (not to scale), and expected pressure profiles for the buffer gas ( $\mathrm{He}$ ) and the $\mathrm{Li}$ vapor (assuming no significant flow in the oven). The length of the Li column and width of the boundary layers are arbitrary on this figure. 


\section{TEMPERATURE PROFILE MEASUREMENTS}

The length over which the $\mathrm{Li}$ vapor extends is estimated from temperature profiles measured with a thermocouple probe along the axis of the heat-pipe oven with and without $\mathrm{Li}$. The profile without $\mathrm{Li}$ in the oven (Fig. 2) shows that the temperature is decreasing away from the oven center, due to heat conduction along the oven wall and along the stainless steel probe housing itself. In contrast, the profiles measured with $\mathrm{Li}$ in the oven and with comparable heating power exhibit a relatively constant temperature in the center of the oven, followed by a rapid drop toward the end of the oven. The Li takes the heat from the center of the oven upon evaporation ( $\mathrm{Li}$ enthalpy of evaporation: $19 \mathrm{~J} / \mathrm{g}$ ), the vapor transports it towards the end of the oven where it is released upon condensation of the vapor on the wick. In this oven, the length over which the vapor density drops to $80 \%$ of the center density is estimated from the temperature profiles and the $\mathrm{Li}$ vapor pressure curve to be $L=25 \mathrm{~cm}\left(P_{\text {litut }}=307 \mathrm{~W}\right)$. Note that the temperature of the oven wall measured by a thermocouple placed outside of the oven, and referred to as $T_{t: x}$ hereafter, is about $60^{\circ} \mathrm{C}$ higher than that measured inside the oven.

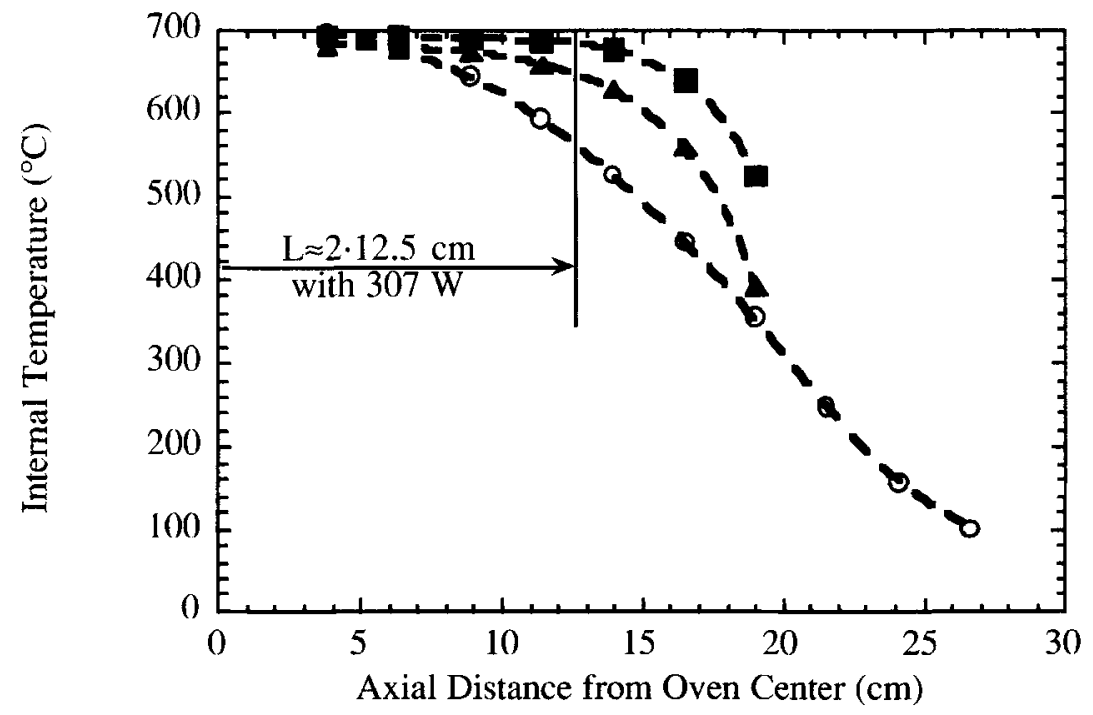

Figure 2: Temperature profiles along the oven axis without $\mathrm{Li}$ in the oven and $P_{\text {hrut }}=250 \mathrm{~W}$ (open circles), and with $\mathrm{Li}$ in the oven and $P_{\text {herm }}=265 \mathrm{~W}$ (filled triangles) and $P_{\text {hest }}=307 \mathrm{~W}$ (filled squares). The lines are drawn to guide the eye. The Li column length defined as the length over which the density $n_{i}$ drops by $20 \%$ (according to the $\mathrm{Li}$ vapor pressure curve) is $L=25 \mathrm{~cm}$ with $P_{\text {her }}=307 \mathrm{~W}$. 


\section{NEUTRAL DENSITY MEASUREMENTS}

In this section two diagnostics for measuring the line integrated $\mathrm{Li}$ neutral density $n_{n} L$ are presented. Assuming a length $L$ of $25 \mathrm{~cm}$, estimates for the average neutral density are obtained.

\section{1 uv absorption}

A low energy $(<1 \mathrm{~mJ})$ laser pulse of $5.83 \mathrm{eV}$ (uv) photons (frequency quintupled Nd:YAG, nanosecond laser pulse) is sent along the heat-pipe oven axis. It ionizes the $\mathrm{Li}$ vapor through a single-photon process ( $\mathrm{Li}$ ionization potential: $5.392 \mathrm{eV}$ ). The product $n_{t} L$ is obtained by measuring the ratio of the transmitted to incident uv energy:

$$
\mathrm{n}_{0} \cdot \mathrm{L}=-\frac{1}{\sigma} \cdot \ln \left(\frac{\mathrm{E}_{\text {transmitted }}}{\mathrm{E}_{\text {incident }}}\right)
$$

where $\sigma=1.8 \times 10^{-18} \mathrm{~cm}^{-2}$ is the ionization cross-section. ${ }^{7}$ For this measurement, the incident and transmitted energies are monitored by photodiodes, and their ratio calculated for each shot. Figure 3 shows the values of $n_{1} L$ measured as function of the oven external temperature. A value of $5.29 \times 10^{16} \mathrm{~cm}^{-2}$ is obtained with $T_{e: 1}=749^{\circ} \mathrm{C}$, corresponding to $n_{t}=2.12 \times 10^{15} \mathrm{~cm}^{-3}$ for $L=25 \mathrm{~cm}$. At this temperature (or density) the fractional uv energy absorbed is only $9.5 \%$.

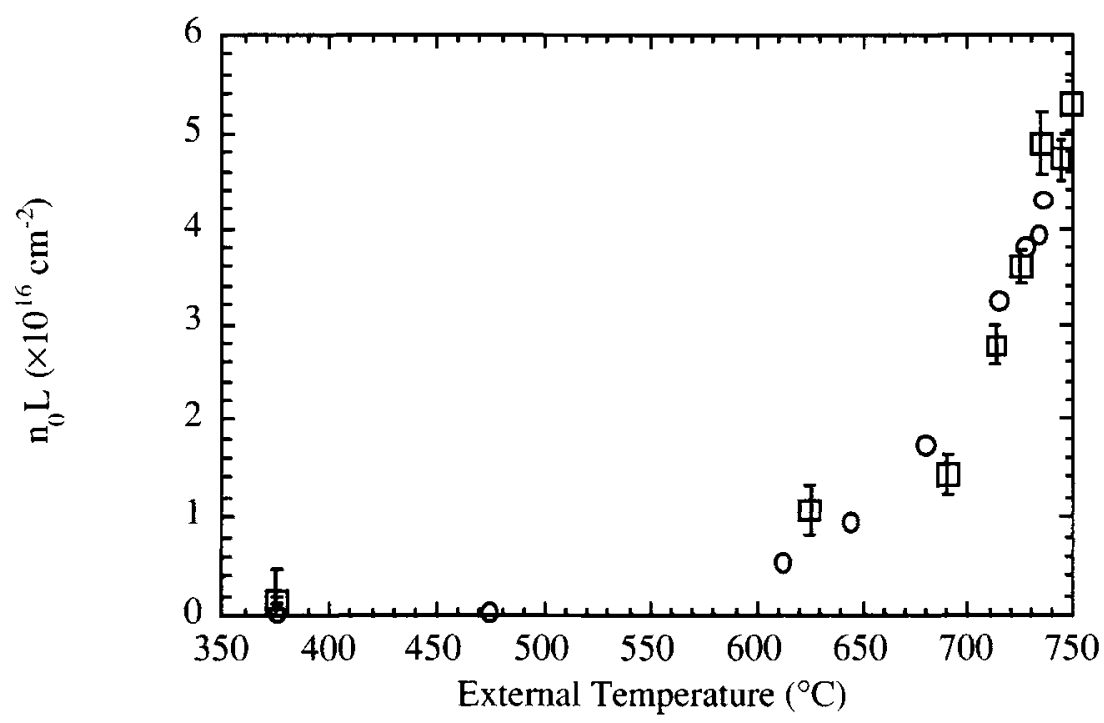

Figure 3: Neutral density measurement. Values of $n_{i} L$ as measured by the uv absorption method (squares) and by the hook method (circles). Assuming $L=25 \mathrm{~cm}$ (see Fig. 2), both method show that $n_{t} \approx 2 \times 10^{15} \mathrm{~cm}^{-3}$ with $T_{e . x t} \approx 750^{\circ} \mathrm{C}$. 


\subsection{Hook method}

Lithium, as do other alkali metals, exhibits a strong transition line in the visible frequency range from its ground state to the first excited state ( $2 \mathrm{~s}$ to $2 \mathrm{p}$ state, $J=1 / 2 \rightarrow I^{\prime}=3 / 2$ at $\lambda=670.78 \mathrm{~nm}$, and $J=1 / 2 \rightarrow I^{\prime}=1 / 2$ at $\lambda=670.79 \mathrm{~nm}$ ). However for the experiments described here, the two Li transitions can be considered as one at $\lambda=670.785 \mathrm{~nm}$. The susceptibility $\chi$ associated with this atomic transition significantly modifies the index of refraction of the Li vapor in the vicinity of $\lambda_{i j}$, even at relatively low density $\left(n_{i 1} \approx 10^{15} \mathrm{~cm}^{-3}\right)$. The corresponding dispersion is used to measure the neutral vapor density and as a diagnostic for the plasma density (see Sect. 6).

The oven is placed in one arm of a Mach-Zehnder white light interferometer. The interferometer light is sent to a stigmatic, $\mathrm{f} / 4.27 \mathrm{~cm}$ spectrograph to observe the dispersion $\left(n(\lambda)=(1+\chi(\lambda))^{1 / 2}\right)$ in the vicinity of the neutral Li line at $670.785 \mathrm{~nm}$ (hook method," see Fig. 4). The evaluation of the hook interferograms uses the following formula:

$$
N_{i} \cdot L=\frac{\pi K}{r_{0} \lambda_{i j}^{3} f_{i j}} \Delta_{i j}^{2}
$$

where $N_{i}$ is the population of the lower level $i, K=p \lambda^{\prime} / \Delta \lambda$ is the hook-interferogram constant, $r_{i 1}=2.82 \times 10^{-15} \mathrm{~m}$ is the classical electron radius, and $f_{i,}$, and $\Delta_{i j}$ are the oscillator strength $\left(f_{i j}=0.75\right)$, and the hook separation of the $i \rightarrow j$ transition. The transition originates from the ground state of the $\mathrm{Li}$ atom. The thermal energy of the $\mathrm{Li}$ atoms is low $\left(k_{B} T_{L i}<<h v_{i j}\right)$, and the population of the ground state is therefore a very good approximation for the vapor density $n_{t}=N_{i}$. The measured values of $n_{o} L$ obtained by this method are shown on Fig. 3 as a function of the oven external temperature. A value of $4.27 \times 10^{16} \mathrm{~cm}^{-2}$ is obtained with $T_{\text {rrt }}=736^{\circ} \mathrm{C}$, corresponding to $n_{t}=1.71 \times 10^{15} \mathrm{~cm}^{-3}$ for $L=25 \mathrm{~cm}$. The values of $n_{v} L$ obtained by the hook method are in very good agreement with the value obtained by the uv absorption method.

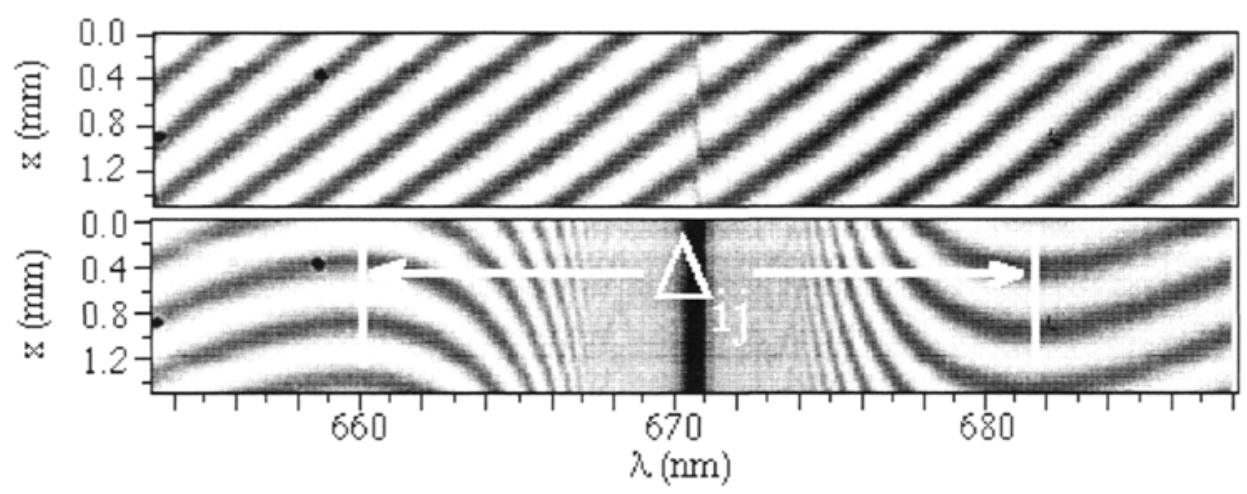

Figure 4: Interferogram (hook method ${ }^{*}$ ), observed in the plane of the imaging spectrograph, showing the hooks created by the dispersion associated with the $2 s \rightarrow 2 p$ transition of the $\mathrm{Li}$ atom $\left(\lambda_{i}=670.785 \mathrm{~nm}\right)$. Top picture: $T_{p r y}=376^{\circ} \mathrm{C}$, there is a negligible density of $\mathrm{Li}$ in the oven. Bottom picture: $T_{e x \mathrm{x}}=715^{\circ} \mathrm{C}$, the measure of $\Delta_{i j}$ (distance between the hooks, in $\mathrm{nm}$ ) yields $n_{t} L=3.2 \times 10^{16} \mathrm{~cm}^{-2}$, which corresponds to $n_{t j}=1.3 \times 10^{15} \mathrm{~cm}^{-3}$, assuming $\mathrm{L}=25 \mathrm{~cm}$. 


\section{LITHIUM PLASMA}

Changes in neutral and/or plasma density modify the dielectric constant $\mathrm{n}$ of the Li vapor and are measured by interferometry. The relative dielectric constant $\varepsilon_{r}$ at frequency $\omega_{i}$ near an atomic transition at frequency $\omega_{i j}$ of a partially ionized gas is given by:

$$
\varepsilon_{\mathrm{r}}=1+\frac{\mathrm{N}_{\mathrm{i}} \mathrm{e}^{2}}{\varepsilon_{0} \mathrm{~m}_{\mathrm{e}}} \sum_{\mathrm{j}} \mathrm{f}_{\mathrm{ij}}\left(\omega_{\mathrm{ij}}^{2}-\omega_{0}^{2}-\mathrm{i} \omega_{0} / \tau_{\mathrm{ij}}\right)^{-1}-\frac{\omega_{\mathrm{pe}}^{2}}{\omega_{0}^{2}}
$$

where $\tau_{i j}$ is the lifetime of the upper state $j$ of the $i \rightarrow j$ transition. It includes a contribution from the resonant transitions (change in $N_{i}$ ) from the lower state $i$ of the neutral atoms to the possible upper states $j$ of the transition (see Section 5.2), and a contribution from the plasma (change in $n_{\text {. }}$ ). Depending on $\omega$, one or both of the contributions need to be retained. The phase difference $\Delta \Phi$ between light from the two arms of an interferometer arising from the change in index of refraction is given by:

where $n=\left(\varepsilon_{r}\right)^{1 / 2}$.

$$
\Delta \Phi=2 \pi(\mathrm{n}-1) \frac{\mathrm{L}}{\lambda_{0}}
$$

The $\mathrm{Li}$ vapor is ionized by the $20 \mathrm{~ns}$. uv light pulse of an $\mathrm{ArF}$ excimer laser at $193 \mathrm{~nm}(h v=6.43 \mathrm{eV})$. The line integrated plasma density $n L$ is directly measured by $\mathrm{CO}_{2}$ interferometry $\left(\lambda_{0}=10.6 \mu \mathrm{m}\right)$. At this wavelength, only the plasma contribution to

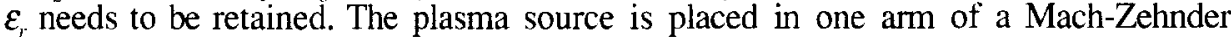
interferometer. The product $n L$ is obtained by measuring the phase shift on the interferometer signal resulting from the change in index of refraction of the recombining plasma:

$$
\mathrm{n}_{\mathrm{e}} \cdot \mathrm{L} \cong-\frac{\Delta \Phi}{\pi} \cdot \lambda_{0} \cdot \mathrm{n}_{\text {crit }} \text { for } \mathrm{n}_{\mathrm{e}} \ll<\mathrm{n}_{\text {crit }}
$$

where $n_{c r i t}$ is the critical plasma density for $\lambda_{\theta}: n_{c r i t}=\left(2 \pi c / \lambda_{i}\right)^{2} \cdot \varepsilon_{i} m_{e} / e^{2}=9.9 \times 10^{18} \mathrm{~cm}^{-3}$. Figure 5 shows the plasma density measured by $\mathrm{CO}_{2}$ interferometry (assuming $L=25 \mathrm{~cm}$ ) as a function of the uv energy incident on the plasma. The maximum density of $1.27 \times 10^{14} \mathrm{~cm}^{-3}$ is limited by the uv energy reaching the $\mathrm{Li}$ vapor. The $\mathrm{CO}_{2}$ laser pulse is coupled into the oven by grazing reflection $(\approx 70 \mathrm{deg}$.) upon a quartz window. When traversing this window about half of the ionizing laser pulse energy is lost by reflection. The time for the plasma to drop by a factor of two, because of recombination and possible diffusion, is about $12 \mu \mathrm{s}$.

The average plasma density $n_{e}$ is estimated from the absorbed uv energy and from the illuminated volume since every photon absorbed by an atom creates one free electron. About $6.6 \%$ of uv energy is absorbed over a $0.67 \mathrm{~cm}^{2}$ beam cross section and $L=25 \mathrm{~cm}$. The average plasma density calculated by this method is also plotted on Fig. 5, and shows a good agreement with the interferometry results. Note that for this measurement the uv energies are averaged over a large number of laser pulses by a calorimeter. Shot-to-shot variation of the uv energy $( \pm 10 \%)$ is responsible for the variations in the plasma density observed by interferometry. The neutral density necessary for a $6.6 \%$ uv absorption is $1.5 \times 10^{15} \mathrm{~cm}^{-3}$; the fractional ionization is therefore about $12 \%$. 


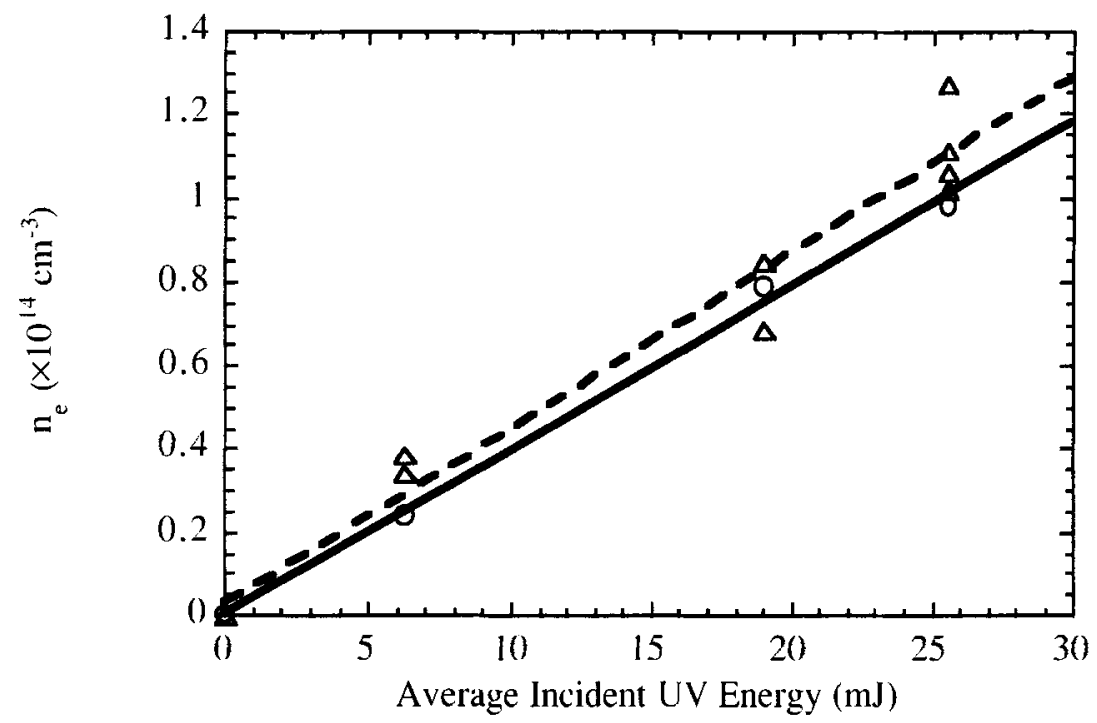

Figure 5: Plasma density as measured by $\mathrm{CO}_{2}$ interferometry (triangles, dashes line), and by absorption of the uv ionizing laser pulse energy (circles, continuous line) as a function of the energy incident upon the $\mathrm{Li}$ vapor. The neutral density $n_{t}$ is $1.52 \times 10^{15} \mathrm{~cm}^{-3}$, and the uv absorption is $6.6 \%$.

Since the plasma is obtained by ionization of the neutrals, the plasma density can be inferred from the variation of the neutral Li density, i.e., by interferometry on the Li neutrals near $\lambda_{i j}=670.785 \mathrm{~nm}$ (see section 5.2). The wavelength at which interferometry is performed can be chosen to observe a suitable number of fringes. An example of the fringe shift observed simultaneously by $\mathrm{CO},\left(\lambda_{i}=10.6 \mu \mathrm{m}\right)$ and by helium neon $\left(\lambda_{n}=632.8 \mathrm{~nm}\right)$ interferometry is shown on Fig. 6 . At $\lambda_{r}=10.6 \mu \mathrm{m}$, only the plasma contribution to $\varepsilon_{r}$ needs to be retained, whereas at $\lambda_{l}=632.8 \mathrm{~nm}$ both the neutrals and the plasma contribute to $\varepsilon_{r}$. The average plasma density obtained at these two wavelengths are $0.42 \times 10^{14} \mathrm{~cm}^{-3}$, and $1.0 \times 10^{14} \mathrm{~cm}^{-3}$ respectively, for a neutral density of $7.6 \times 10^{14} \mathrm{~cm}^{-3}$ and an incident uv fluence of $30 \mathrm{~mJ} / \mathrm{cm}^{-2}$. The value of $n_{e}$ obtained from the uv absorption (3.1\%) is $0.37 \times 10^{14} \mathrm{~cm}^{-3}$ and is in good agreement with the $\mathrm{CO}_{2}$ interferometry value. Note that the uv pulse spot shape is not uniform and may account for the discrepancy between the interferometry results. The uv absorption and the $\mathrm{CO}_{2}$ interferometry yield volume integrated values of the plasma density, whereas the helium-neon pencil beam only samples a cord along the oven axis. Interferometry on the neutrals at the helium-neon laser wavelength is suitable for the 1meter plasma source $\left(n_{c} \approx 2-4 \times 10^{14} \mathrm{~cm}^{-3}\right)$ since the fringe shift is expected to be about ten times larger than the one observed with the prototype. 


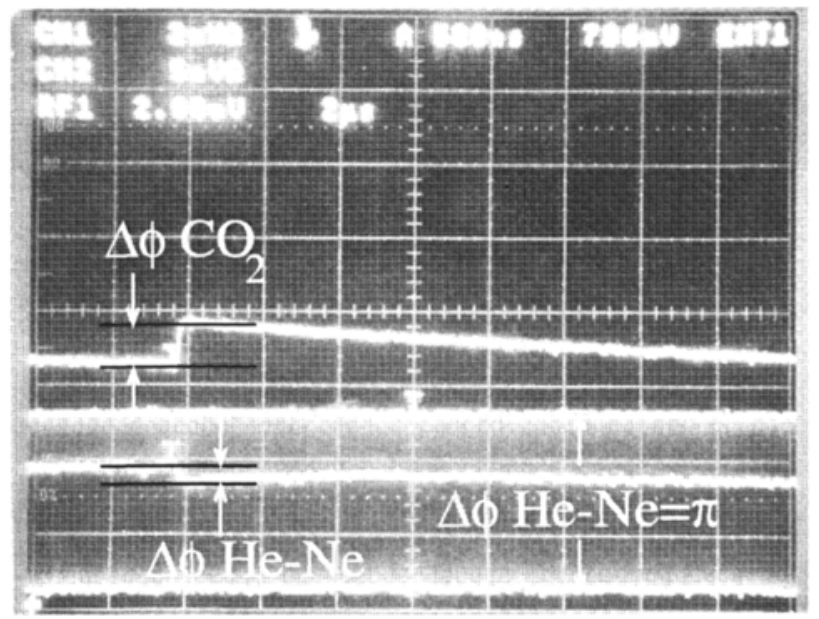

Figure 6: Oscilloscope traces obtained simultaneously from the $\mathrm{CO}_{2}$ interferometry (top trace) and the helium-neon interferometry (bottom trace).

The maximum plasma density obtained with this prototype is at $T_{t \rightarrow 1}=734^{\circ} \mathrm{C}$ $\left(n_{t} \approx 2.0 \times 10^{1.5} \mathrm{~cm}^{-2}\right.$ over $L=25 \mathrm{~cm}$, Fig. 3) with a uv fluence of $35 \mathrm{~mJ} / \mathrm{cm}^{-2}$, leading to an average plasma density of $n_{c}=2.6 \times 10^{14} \mathrm{~cm}^{-3}$, and a fractional ionization $n_{r} / n_{t}$ of $13 \%$. This value is limited only by the available uv fluence.

\section{SUMMARY}

A lithium vapor density in the $2 \times 10^{15} \mathrm{~cm}^{-3}$ range is obtained in a heat-pipe oven. The length of the neutral Li column is about $25 \mathrm{~cm}$. The values for the neutral density obtained by uv absorption and by the hook method are in very good agreement. The vapor is ionized by uv light (1-photon process). The line integrated plasma density is measured by $\mathrm{CO}_{2}$ laser interferometry on the plasma electrons, by visible interferometry on the $\mathrm{Li}$ neutrals (near the $\lambda_{i /}=670.785 \mathrm{~nm}$ transition from the $\mathrm{Li}$ ground state), and by uv absorption. The maximum plasma density is $2.6 \times 10^{14} \mathrm{~cm}^{-3}$, limited only by the maximum uv fluence available. The time for the plasma density to drop by a factor of two is about $12 \mu \mathrm{s}$. Visible interferometry on the neutrals (heliumineon laser, $\lambda_{i}=632.8 \mathrm{~nm}$ ) provides a simple diagnostic for $n_{\text {ond }}$ and will be implemented in the SLAC E-157 experiment. The plasma density will be optimized for the acceleration experiment by changing the delay between the ionizing laser pulse and the electron bunch. The plasma column length will be adjusted by changing the oven heating power. The 1-meter long plasma source is presently being assembled. This source differs from the prototype only by its length, therefore similar neutral/plasma densities should be obtained. A uv fluence of $200 \mathrm{~mJ} / \mathrm{cm}^{-2}$ incident upon a vapor density of $6 \times 10^{14} \mathrm{~cm}^{-3}$ is required to produce a $2 \times 10^{14} \mathrm{~cm}^{-3}$ plasma density with a $10 \%$ drop along the 1-meter plasma. The variation of the plasma density over the length of the oven due to the depletion of the ionizing pulse energy can be minimized by sending the pulse back through the oven. Double passing the oven with the uv beam would also almost double the plasma density. 


\section{ACKNOWLEDGMENTS}

The authors would like to thank Prof. P. Muntz from USC for lending the excimer laser. This work is supported by the US Department of Energy under Grants No. DE-FG03-92ER40727 and No. DE-FG03-92ER40745, and by the National Science Foundation under Grants No. ECS-9632735 and No. ECS-9617089.

\section{REFERENCES}

' D. Gordon, K.C. Tzeng, C.E. Clayton, A.E. Dangor, V. Malka, K.A. Marsh, A. Modena, W.B. Mori, P. Muggli, Z. Najmudin, D. Neely, C. Danson, and C. Joshi, "Observation of electron energies beyond the linear dephasing limit from a laser-excited relativistic plasma wave," Phys. Rev. 80(10), 2133 (1998), and papers in this working group.

${ }^{2}$ C.E. Clayton, K.A. Marsh, A. Dyson, M. Everett, A. Lal, W.P. Leemans, R. Williams, and C. Joshi, "Ultrahigh-Gradient Acceleration of injected electrons by Laser-excited relativistic electron plasma wave," Phys. Rev. Lett. 70(1), 37 (1993).

${ }^{3}$ T. Katsouleas, S. Lee, S. Chattopadhyay, W.L. Leemans, R. Assmann, P. Chen, F.J. Decker, R. Iverson, T. Kotseroglou, P. Raimondi, T. Raubenheimer, S. Ronki, R.H. Siemann, D. Walz, D. Whittum, C.E. Clayton, C. Joshi, K. Marsh, W.B. Mori, and G. Wang, "A proposal for a $1 \mathrm{GeV}$ plasma-wakefield acceleration at SLAC." Proceedings of the Particle Accelerator Conference, Vancouver, Canada, May 1997.

${ }^{4}$ D. Gordon, C.E. Clayton, and C. Joshi, "Collisional ionization of gases by $\mathrm{GeV}$ beams," presented at the Seventh Advanced Accelerator Concept Workshop, Lake Tahoe CA, (1996).

${ }^{5}$ CRC Handbook of Chemistry and Physics, 72nd Ed., CRC Press Inc, (1991).

"C.R. Vidal and J. Cooper, "Heat-pipe oven: a new, well-defined metal vapor device for spectroscopic measurements," J. Appl. Phys. 40(8), 3370 (1969).

${ }^{7}$ G.V. Marr, "Photoionization process in gases," Academic Press. (1967).

${ }^{8}$ W.C. Marlow, "Hakenmethode," Applied Optics 6(10), 1715 (1967). 